\title{
Correction to: Radiological approach for the newly incorporated T staging factor, depth of invasion (DOI), of the oral tongue cancer in the 8th edition of American Joint Committee on Cancer (AJCC) staging manual: assessment of the necessity for elective neck dissection
}

\section{Akira Baba $^{1} \cdot$ Kazuhiko Hashimoto $^{2} \cdot$ Reina Kayama $^{1} \cdot$ Hideomi Yamauchi $^{1} \cdot$ Koshi Ikeda $^{1} \cdot$ Hiroya Ojiri $^{1}$}

Published online: 6 October 2020

(c) Japan Radiological Society 2020

\section{Correction to:}

Japanese Journal of Radiology (2020) 38:821-832

https://doi.org/10.1007/s11604-020-00982-w
- Extrinsie tongue musele invasion.

The corrected Table 1 is given in this Correction.

In the original publication of this paper Table 1 has been

mistakenly published without the entry below:

Table 1 T categorization of the oral cavity cancer in the American Joint Committee on Cancer (AJCC) Cancer Staging Manual, 8th edition

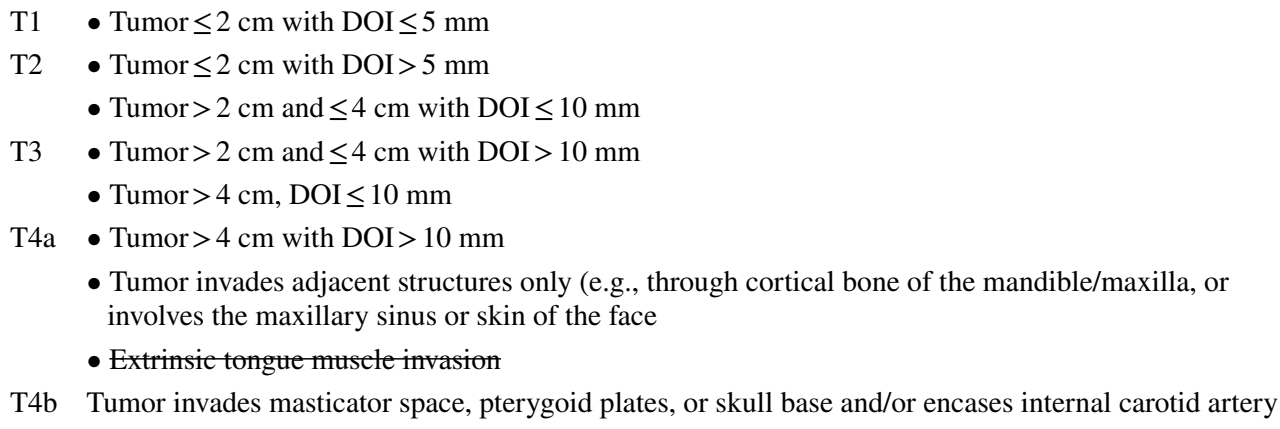

The item with line-through was excluded from T categorization in AJCC Cancer Staging Manual, 8th edition
The original article can be found online at https://doi.org/10.1007/ s11604-020-00982-w.

Akira Baba

akirababa@jikei.ac.jp

1 Department of Radiology, The Jikei University School of Medicine, 3-25-8 Nishi-Shimbashi, Minato-ku,

Tokyo 105-8461, Japan
Publisher's Note Springer Nature remains neutral with regard to jurisdictional claims in published maps and institutional affiliations. 